\title{
ON THE ORDER OF AUTOMORPHISM GROUPS OF KLEIN SURFACES
}

\author{
by J. J. ETAYO GORDEJUELA
}

(Received 18 November, 1983)

1. Introduction. A problem of special interest in the study of automorphism groups of surfaces are the bounds of the orders of the groups as a function of the genus of the surface.

May has proved that a Klein surface with boundary of algebraic genus $p$ has at most $12(p-1)$ automorphisms [9].

In this paper we study the highest possible prime order for a group of automorphisms of a Klein surface. This problem was solved for Riemann surfaces by Moore in [10]. We shall use his results for studying the Klein surfaces that are not Riemann surfaces. The more general result that we obtain is the following: if $X$ is a Klein surface of algebraic genus $p$, and $G$ is a group of automorphisms of $X$, of prime order $n$, then $n \leqslant p+1$.

2. Preliminaries. A Klein surface $X$ is a surface with or without boundary, with an open covering $U=\left\{U_{i}\right\}_{i \in I}$ that fulfills the following two conditions:

(i) For each $U_{i} \in \mathcal{U}$ there exists a homeomorphism $\phi_{i}$ from $U_{i}$ onto an open subset of $\mathbb{C}$.

(ii) If $U_{i}, U_{i} \in \mathcal{U}, U_{i} \cap U_{j} \neq \varnothing$, then $\phi_{i} \phi_{j}^{-1}$ is an analytic or anti-analytic application defined in $\phi_{j}\left(U_{i} \cap U_{j}\right)$.

An automorphism of the surface is a homeomorphism $f: X \rightarrow X$ such that $\phi_{i} f \phi_{j}^{-1}$ is analytic or anti-analytic.

Orientable Klein surfaces without boundary are Riemann surfaces.

A non-orientable Klein surface $X$ with topological genus $g$ and $k$ boundary components has algebraic genus $p=g+k-1$; if $X$ is orientable with boundary, its algebraic genus is $p=2 g+k-1$.

Klein surfaces and their automorphisms may be studied by means of non-Euclidean crystallographic groups (NEC groups). An NEC group is a discrete subgroup of isometries of the non-Euclidean plane with compact quotient space. NEC groups include reversing orientation isometries, reflections and glide-reflections.

NEC groups are classified according to their signatures. The signature of an NEC group is of the form

$$
\left(\mathrm{g}, \pm,\left[m_{1}, \ldots, m_{r}\right],\left\{\left(n_{11}, \ldots, n_{1 s_{1}}\right), \ldots,\left(n_{k 1}, \ldots, n_{k s_{k}}\right)\right\}\right) .
$$

The number $g$ is the genus, the $m_{i}$ are the proper periods, and the brackets $\left(n_{i 1}, \ldots, n_{i s_{i}}\right)$ are the period-cycles.

The group $\Gamma$ with signature $(*)$ has a presentation given by generators

(i) $x_{i}, i=1, \ldots, r$

(ii) $e_{i}, i=1, \ldots, k$

Glasgow Math. J. 26 (1985) 75-81. 
(iii) $c_{i j}, i=1, \ldots, k, j=0, \ldots, s_{i}$

(iv) (with sign ' + ') $a_{j}, b_{j}, j=1, \ldots, g$ (with sign ' - ') $d_{j}, j=1, \ldots, g$

and relations

(i) $x_{i}^{m_{i}}=1, i=1, \ldots, r$

(ii) $e_{i}^{-1} c_{i 0} e_{i} c_{i s_{\mathrm{i}}}=1, i=1, \ldots, k$

(iii) $c_{i, j-1}^{2}=c_{i j}^{2}=\left(c_{i, j-1} c_{i j}\right)^{n_{i i}}=1, i=1, \ldots, k, j=1, \ldots, s_{i}$

(iv) (with sign ' + ') $x_{1} \ldots x_{r} e_{1} \ldots e_{k} a_{1} b_{1} a_{1}^{-1} b_{1}^{-1} \ldots a_{\mathrm{g}} b_{\mathrm{g}} a_{\mathrm{g}}^{-1} b_{\mathrm{g}}^{-1}=1$

(with sign '-') $x_{1} \ldots x_{r} e_{1} \ldots e_{k} d_{1}^{2} \ldots d_{\mathrm{g}}^{2}=1$.

The area of a fundamental region for an NEC group $\Gamma$ is given by

$$
|\Gamma|=2 \pi\left(\alpha g+k-2+\sum_{i=1}^{r}\left(1-\frac{1}{m_{i}}\right)+\frac{1}{2} \sum_{i=1}^{k} \sum_{j=1}^{s_{i}}\left(1-\frac{1}{n_{i j}}\right)\right),
$$

where $\alpha=1$ if the sign is '-' and $\alpha=2$ if the sign is ' + '.

The relation between Klein surfaces and NEC groups comes from the following two results:

THEOREM A. [12]. Let $X$ be a Klein surface of topological genus $g, k$ boundary components, and algebraic genus $\geqslant 2$. Then $X$ may be represented as $D / K$, where $D=$ $\{z \in \mathbb{C}, \operatorname{im} z>0\}$ and $K$ is an NEC group with signature $(g, \pm,[-],\{(-), \ldots,(-)\})$ with sign ' + ' if $X$ is orientable, and ' - ' if $X$ is non-orientable.

THEOREM B. [8]. A finite group $G$ is a group of automorphisms of the Klein surface $D / K$ if and only if $G=\Gamma / K$, where $\Gamma$ is an NEC group from which $K$ is a normal subgroup.

We shall establish first of all a result about normal subgroups of NEC groups.

LEMMA 1. Let $\Gamma$ be an NEC group, with signature $\left(g, \pm,\left[m_{1}, \ldots, m_{\mathrm{r}}\right],\{(-), \ldots,(-)\}\right)$, and let $\Gamma_{0}$ be a normal subgroup of $\Gamma$, such that $\left|\Gamma: \Gamma_{0}\right|=N$. If $q_{i}$ is the least integer such that $e_{i}^{q_{i}} \in \Gamma_{0}, i=1, \ldots, k$, and if $c_{10}, \ldots, c_{k 0}$ belong to $\Gamma_{0}$, then the signature of $\Gamma_{0}$ has $\left(N / q_{1}\right)+\ldots+\left(N / q_{k}\right)$ period-cycles, all of them empty.

Proof. If $\Delta$ is an NEC group with empty period-cycles, then the number of them is equal to the number of conjugacy classes of reflections in $\Delta$, as both equal the number of holes in $D / \Delta$. In our case $\Gamma_{0}$ has empty period-cycles, and we need only find the number of conjugacy classes of reflections in $\Gamma_{0}$. We will show that there are $N / q_{i}$ conjugacy classes of reflections in $\Gamma_{0}$ which are conjugate to $c_{i 0}$, for $i=1, \ldots, k$.

The centralizer of $c_{i 0}$ in $\Gamma$ is the abelian group $A_{i}$ generated by $e_{i}$ and $c_{i 0}$ [13]. If $\theta: \Gamma \rightarrow \Gamma / \Gamma_{0}$ is the canonical homomorphism, then as $c_{i 0} \in \Gamma_{0}, \theta\left(A_{i}\right)=\left\{1, e_{i}, \ldots, e_{i}^{a_{i}-1}\right\}$ which has index $N / q_{i}$ in $\Gamma / \Gamma_{0}$. To prove the result we need only show that if $g, h \in \Gamma$ then $g c_{i 0} g^{-1}$ and $h c_{i 0} h^{-1}$ are conjugate in $\Gamma_{0}$ if and only if $\theta(\mathrm{g})$ and $\theta(h)$ lie in the same coset of $\theta\left(A_{i}\right)$ in $\Gamma / \Gamma_{0}$, i.e., $\theta\left(h^{-1} g\right) \in \theta\left(A_{i}\right)$. This result holds for $\theta\left(h^{-1} g\right) \in \theta\left(A_{i}\right)$ is equivalent to $h^{-1} g=\lambda_{0} b$ where $\lambda_{0} \in \Gamma_{0}, b \in A_{i}$, and then $g c_{i 0} g^{-1}=h \lambda_{0} b c_{i 0} b^{-1} \lambda_{0}^{-1} h^{-1}=h \lambda_{0} c_{i 0} \lambda_{0}^{-1} h^{-1}=$ $\gamma_{0} h c_{i 0} b^{-1} \gamma_{0}^{-1} h^{-1}=h \lambda_{0} c_{i 0} \lambda_{0}^{-1} h^{-1}=\gamma_{0} h c_{i 0} h^{-1} \gamma_{0}^{-1}$, where $h \lambda_{0} h^{-1}=\gamma_{0} \in \Gamma_{0}$. 
Thus we have shown that the number of conjugacy classes of reflections in $\Gamma_{0}$ conjugate to $c_{i 0}$ is $N / q_{i}$ and so the number of conjugacy classes of reflections in $\Gamma_{0}$ is $\left(N / q_{1}\right)+\ldots+\left(N / q_{k}\right)$.

3. Prime order groups of automorphisms. Let $X$ be a Klein surface that is not a Riemann surface. Then $X$ may be represented as $D / K$, where $K$ is an NEC group with signature (i) $(g,-,[-],\{-\})$, (ii) $(g,+,[-],\{(-), \ldots,(-)\})$ or (iii) $(g,-,[-]$, $\{(-)$, $\ldots,(-)\}$ ) if $X$ is (i) without boundary, (ii) orientable with boundary, or (iii) nonorientable with boundary.

If $G$ is a group of automorphisms of $X$, with prime order $\neq 2$, then $G=\Gamma / K$, where $\Gamma$ is an NEC group with signature (i) $\left(\gamma,-,\left[\mu_{1}, \ldots, \mu_{r}\right],\{-\}\right)$, (ii) $\left(\gamma,+,\left[\mu_{1}, \ldots, \mu_{r}\right],\{(-)\right.$, $\left.\left.k^{\prime},(-)\right\}\right)$ or (iii) $\left(\gamma,-,\left[\mu_{1}, \ldots, \mu_{r}\right],\{(-), \ldots,(-)\}\right)[3]$.

Let $\Gamma^{+}$and $K^{+}$be the canonical fuchsian subgroups associated to $\Gamma$ and $K$, i.e., the subgroups formed by the elements which preserve orientation, [13]. Then, by [7, Cor. 1], if $t \in \Gamma / K$ has $N$ fixed points, $t \in \Gamma^{+} / K^{+}$has $2 N$ fixed points. We shall denote by $N(t)$ the number of fixed points of $t$.

We shall indicate now the main result obtained by Moore for Riemann surfaces, that -will be used throughout this paper.

LEMma 2 [10]. Let $S$ be a Riemann surface of genus $g$. Let $K$ be the fuchsian group with signature $(\mathrm{g},+,[-],\{-\})$, and $G$ a group of automorphisms of $S$, with prime order $n \neq 2$. Then $G=\Gamma / K$, where $\Gamma$ has signature $\left(\gamma,+,\left[\mu_{1}, \ldots, \mu_{r}\right],\{-\}\right)$; and $n \geqslant g$ only in the next four cases:

(i) $n=2 \mathrm{~g}+1, N(t)=3, \gamma=0$,

(ii) $n=g+1, N(t)=4, \gamma=0$,

(iii) $n=g=3, N(t)=5, \gamma=0$, or

(iv) $n=g, N(t)=2, \gamma=1$,

where $t$ is a generator of the group $G$.

THEOREM 1. Let $X$ be a Klein surface of algebraic genus $p \geqslant 2$. Let $G$ be a group of automorphisms of $X$, of prime order $n$. Then if $X$ is without boundary, or orientable with boundary, $n \leqslant p+1$; and if $X$ is non-orientable with boundary, $n \leqslant p$.

Proof. 1. Let $X$ be non-orientable, without boundary, of genus $g$. Then $X=D / K$, $G=\Gamma / K$, and the signatures of $K$ and $\Gamma$ are $(g,-,[-],\{-\})$ and $\left(\gamma,-,\left[\mu_{1}, \ldots, \mu_{r}\right],\{-\}\right)$. From [13], the subgroups $\Gamma^{+}$and $K^{+}$have signatures, respectively, $\left(\gamma-1,+,\left[\mu_{1}, \mu_{1}, \ldots, \mu_{r}, \mu_{r}\right],\{-\}\right)$ and $(g-1,+,[-],\{-\})$. Also $\left|\Gamma^{+}: K^{+}\right|=n$, and so by Lemma 2 , if $n>g-1$, we must have

(i) $n=2(g-1)+1, N(t)=3, \gamma-1=0$, or

(ii) $n=(g-1)+1, N(t)=4, \gamma-1=0$.

If case (i) holds, by [7, Corollary 1] $t \in \Gamma / K$ would have $3 / 2$ fixed points, impossible. So the highest possible prime $n$, is $n=(g-1)+1=g=p+1$, and then $N(t)=4 / 2=2, \gamma=1$.

This bound is attained for every $p+1$ prime: The group $\Gamma$ with signature 
$(1,-,[p+1, p+1],\{-\})$ fulfills the conditions of [4, Theorem 3.7] and hence there is an epimorphism from $\Gamma$ onto $Z / p+1$ whose kernel has signature $(g,-,[-],\{-\})$. By the relation of areas $[\mathbf{1 3}]$,

so, $g=p+1$.

$$
1+p=\frac{g-2}{1-2+2\left(1-\frac{1}{p+1}\right)}=\frac{g-2}{1-\frac{2}{p+1}}=\frac{(g-2)(p+1)}{p-1}
$$

2. Let $X$ be orientable, with boundary, of algebraic genus $p$. Then $X=D / K$, $G=\Gamma / K$, and the signatures of $\Gamma$ and $K$ are $\left(\gamma,+,\left[\mu_{1}, \ldots, \mu_{r}\right],\left\{(-), k^{\prime},(-)\right\}\right)$ and $(g,+,[-],\{(-), \ldots,(-)\})$. The canonical fuchsian subgroups $\Gamma^{+}$and $K^{+}$have signature $\left(2 \gamma+k^{\prime}-1,+,\left[\mu_{1}, \mu_{1}, \ldots, \mu_{r}, \mu_{r}\right],\{-\}\right)$ and $(2 g+k-1,+,[-],\{-\})$, i.e., the signature of $K^{+}$is $(p,+,[-],\{-\})$.

By Lemma 2, if $n>p$, we must have

(i) $n=2 p+1, N(t)=3,2 \gamma+k^{\prime}-1=0$, or

(ii) $n=p+1, N(t)=4,2 \gamma+k^{\prime}-1=0$.

As before, the case (i) is impossible, and the highest possible prime $n$ is $n=p+1$, and then $N(t)=4 / 2=2,2 \gamma+k^{\prime}-1=0$. As $k^{\prime} \neq 0$, it is $\gamma=0, k^{\prime}=1$.

3. Let $X$ be non-orientable, with boundary, of algebraic genus $p$. Then $X=D / K$, $G=\Gamma / K$, and the signatures of $\Gamma$ and $K$ are $\left(\gamma,-,\left[\mu_{1}, \ldots, \mu_{r}\right],\{(-), \ldots,(-)\}\right)$ and $(g,-,[-],\{(-), \ldots,(-)\})$. The subgroups $\Gamma^{+}$and $K^{+}$have signatures $\left(\gamma+k^{\prime}-\right.$ $\left.1,+,\left[\mu_{1}, \mu_{1}, \ldots, \mu_{r}, \mu_{r}\right],\{-\}\right)$ and $(p,+,[-],\{-\})$. By Lemma 2 , if $n>p$, it must be

(i) $n=2 p+1, N(t)=3, \gamma+k^{\prime}-1=0$, or

(ii) $n=p+1, N(t)=4, \gamma+k^{\prime}-1=0$.

As before, the case (i) is impossible; but as $\gamma \geqslant 1, k^{\prime} \geqslant 1, \gamma+k^{\prime}-1$ is different from 0 , and so the case (ii) is also impossible. Hence in no case is $n>p$.

Let us see now when $n=p$. By Lemma 2, we must have

(i) $n=p=3, N(t)=5, \gamma+k^{\prime}-1=0$, or

(ii) $n=p, N(t)=2, \gamma+k^{\prime}-1=1$.

The case (i) is again impossible, and thus the only possible case is $n=p, N(t)=2 / 2=1$, $\gamma=k^{\prime}=1$.

We have obtained the bounds of the order as a function of the algebraic genus of the surface. We shall calculate now which are the values of the topological genus and boundary components that attain these bounds.

In the non-orientable surfaces without boundary the algebraic and topological genera are mutually determined. So we have seen that every prime $g$ attains the bound.

Let us now study the surfaces with boundary.

Proposmion 1. Let $X$ be an orientable Klein surface with boundary of algebraic genus p. (1) If $p+1$ is prime, there is a group of automorphisms of $X$, of order $p+1$, if and only if $X$ has 1 or $p+1$ boundary components, and topological genus $p / 2$ and 0 , respectively. (2) If $p$ is prime, there is a group of automorphisms of $X$, or order $p$, if and only if $X$ has 2 or $p+1$ 
boundary components, and topological genus ( $p-1) / 2$ and 0 , respectively. (3) Otherwise, any automorphisms group of $X$ with prime order has order smaller than $p$.

Proof. Let $n$ be the order of the group, say $G$. By Theorem 1 , if $n>p$, we have $n=p+1$. Also $X=D / K, G=\Gamma / K$, and $\Gamma$ and $K$ have signatures $\left(0,+,\left[\mu_{1}, \ldots, \mu_{r}\right],\{(-)\}\right)$ and $(g,+,[-],\{(-), \ldots,(-)\})$, with $2 g+k-1=p$. Hence by Lemma $1, k \mid p+1$, and so $k=1$ or $k=p+1$.

If $k=1, K$ has signature $(p / 2,+,[-],\{(-)\})$. The group $\Gamma$ with signature $(0,+,[p+$ $1, p+1],\{(-)\})$ fulfills the conditions of [5, Theorem 3.5] and hence there is an epimorphism $\theta$ from $\Gamma$ onto $Z / p+1$ whose kernel has signature $(g,+,[-],\{(-)\})$. By the relation of areas,

$$
p+1=\frac{2 g-1}{-1+2(1-(1 / p+1))}=\frac{(2 g-1)(p+1)}{p-1}
$$

thus $g=p / 2$ and so $\operatorname{ker} \theta=K$.

If $k=p+1, K$ has signature $(0,+,[-],\{(-), \stackrel{p+1}{\cdots},(-)\})$. Let $\Gamma$ be the group with signature $(0,+,[p+1, p+1],\{(-)\})$. The epimorphism $\theta$ from $\Gamma$ onto $Z / p+1$ given by $\theta\left(x_{1}\right)=\overline{1}, \theta\left(x_{2}\right)=\bar{p}, \theta\left(e_{1}\right)=\theta\left(c_{10}\right)=\overline{0}$, verifies that its kernel has signature $(g,+,[-]$, $\{(-), \ldots,(-)\})$, and

$$
p+1=\frac{2 g+p-1}{-1+2(1-(1 / p+1))}=\frac{(2 g+p-1)(p+1)}{p-1}
$$

thus $g=0$, and so $\operatorname{ker} \theta=K$.

Let us see now when $n=p$, prime. By Lemma 2 , we have $2 \gamma+k^{\prime}-1=1$, and so $\gamma=0, k^{\prime}=2$. Thus $\Gamma$ has signature $\left(0,+,\left[\mu_{1}, \ldots, \mu_{r}\right],\{(-)(-)\}\right)$. By Lemma $1, k=$ $k_{1}+k_{2}$, where $k_{\mathrm{i}} \mid p$, and hence $k=2, k=p+1$, or $k=2 p$. As $2 g+k-1=p, k=2 p$ is impossible.

If $k=2, K$ has signature $((p-1) / 2,+,[-],\{(-)(-)\})$. Let $\Gamma$ be the group with signature $(0,+,[p],\{(-)(-)\})$. The epimorphism $\theta$ form $\Gamma$ onto $Z / p$ given by $\theta\left(x_{1}\right)=\overline{1}$, $\theta\left(e_{1}\right)=\theta\left(e_{2}\right)=\overline{(p-1) / 2}, \theta\left(c_{10}\right)=\theta\left(c_{20}\right)=\overline{0}$, verifies that its kernel has signature $(g,+,[-]$, $\{(-)(-)\})$, and

$$
p=\frac{2 g}{1-(1 / p)}=\frac{2 g p}{p-1}
$$

thus $g=(p-1) / 2$ and so $\operatorname{ker} \theta=K$.

If $k=p+1, K$ has signature $(0,+,[-],\{(-), \ldots, 1,(-)\})$. Let $\Gamma$ be the group with signature $(0,+,[p],\{(-)(-)\})$. The epimorphism $\theta$ from $\Gamma$ onto $Z / p$ given by $\theta\left(x_{1}\right)=\overline{1}$, $\theta\left(e_{1}\right)=\overline{p-1}, \theta\left(e_{2}\right)=\theta\left(c_{10}\right)=\theta\left(c_{20}\right)=\overline{0}$, verifies that its kernel has signature $(g,+,[-]$, $\{(-), \stackrel{p+1}{\cdots},(-)\})$, and

$$
p=\frac{2 g+p-1}{1-(1 / p)}=\frac{(2 g+p-1) p}{p-1}
$$

thus $g=0$, and so $\operatorname{ker} \theta=K$. 
Proposition 2. Let $X$ be a non-orientable Klein surface with boundary, of algebraic genus $p$. (1). If $p$ is prime, there is a group of automorphisms of $X$, of order $p$, if and only if $X$ has 1 or $p$ boundary components, and topological genus $p$ and 1, respectively. (2) Otherwise, any automorphisms group of $X$ with prime order has order smaller than $p$.

Proof. Let $n$ be the order of the group, say $G$. By Theorem 1 , if $n=p, X=D / K$, $G=\Gamma / K$, and $\Gamma$ and $K$ have signatures $\left(1,-,\left[\mu_{1}, \ldots, \mu_{r}\right],\{(-)\}\right)$ and $(g,-,[-],\{(-)$, $\ldots,(-)\})$, with $g+k-1=p$. Hence by Lemma $1, k \mid p$, and so $k=1$ or $k=p$.

If $k=1, K$ has signature $(p,-,[-],\{(-)\})$. The group $\Gamma$ with signature $(1,-,[p]$, $\{(-)\})$ fulfills the conditions of [5, Theorem 3.6] and hence there is an epimorphism $\theta$ from $\Gamma$ onto $Z / p$ whose kernel has signature $(g,-,[-],\{(-)\})$. By the relation of areas,

$$
p=\frac{g-1}{1-(1 / p)}=\frac{(g-1) p}{p-1}
$$

thus, $g=p$, and so $\operatorname{ker} \theta=K$.

If $k=p, K$ has signature $(1,-,[-],\{(-), \ldots,(-)\})$. Let $\Gamma$ be the group with signature $(1,-,[p],\{(-)\})$. The epimorphism $\theta$ from $\Gamma$ onto $Z / p$ given by $\theta\left(x_{1}\right)=\overline{1}$, $\theta\left(d_{1}\right)=\overline{(p-1) / 2}, \theta\left(e_{1}\right)=\theta\left(c_{10}\right)=\overline{0}$, verifies that its kernel has signature $(g,-,[-]$, $\{(-), \ldots,(-)\})$, and

$$
p=\frac{g+p-2}{1-(1 / p)}=\frac{(g+p-2) p}{p-1}
$$

thus $g=1$, and so $\operatorname{ker} \theta=K$.

4. Real algebraic curves. These results may be rewritten in terms of real algebraic curves, as follows:

COROLlaRY 1. Let $V$ be an irreducible real algebraic curve of genus $g \geqslant 2$, and let $V_{c}$ be its complexification. If $V_{\mathbb{C}} \backslash V$ is not connected, then,

1. If $g+1$ is prime, there is a group of automorphisms of $V$, of order $g+1$, if and only if $V$ is connected or has $g+1$ connected components.

2. If $g$ is prime, there is a group of automorphisms of $V$, of order $g$, if and only if $V$ has 2 or $g+1$ connected components.

3. Otherwise, any automorphisms group of $V$ with prime order has order smaller than $g$.

COROLlaRY 2. Let $V$ be an irreducible real algebraic curve of genus $g \geqslant 2$, and let $V_{\mathbb{C}}$ be its complexification. If $V_{\mathbb{C}} \backslash V$ is connected, then

1. If $g$ is prime, there is a group of automorphisms of $V$, of order $g$, if and only if $V$ is connected or has g connected components.

2. Otherwise, any automorphisms group of $V$ with prime order has order smaller than $g$.

Proof of Both Corollaries. By $[\mathbf{1 , 2}]$ there is a functorial equivalence between the category of compact Klein surfaces with boundary, and that of irreducible real algebraic curves. So, each compact Klein surface with $k$ boundary components has associated an 
irreducible real algebraic curve that admits a bounded smooth model with $k$ connected components, and conversely. cation.

From [11], the surface is orientable if and only if the curve disconnects its complexifi-

Further, the groups of automorphisms of the curve and of the surface are isomorphic [6].

Hence, it suffices to rewrite Theorem 1 and Propositions 1 and 2, in this language.

This paper forms part of the doctoral thesis of the author, directed by Professor E. Bujalance. I express him my acknowledgement. Thanks are also due to the referee for his suggestions on shortening the proof of Lemma 1.

\section{REFERENCES}

1. N. L. Alling, Real elliptic curves. Notes of Math. 54, (North-Holland, 1981).

2. N. L. Alling and N. Greenleaf, Foundations of the theory of Klein surfaces. Lecture Notes in Math. 219, (Springer-Verlag, 1971).

3. E. Bujalance, Normal subgroups of NEC groups, Math. Z. 178 (1981) 331-341.

4. E. Bujalance, Cyclic groups of automorphisms of compact non-orientable Klein surfaces without boundary, Pacific J. of Math. 109 (1983) 279-289.

5. E. Bujalance, Automorphisms groups of compact Klein surfaces with one boundary component, (to appear).

6. E. Bujalance and J. M. Gamboa, Automorphisms groups of algebraic curves of $\mathbb{R}^{n}$ of genus 2, Archiv der Math. 42 (1984) 229-237.

7. J. J. Etayo, NEC subgroups in Klein surfaces. Bol. Soc. Mat. Mex. (to appear).

8. C. L. May, Large automorphism groups of compact Klein surfaces with boundary, Glasgow Math. J. 18 (1977) 1-10.

9. C. L. May, A bound for the number of automorphisms of a compact Klein surface with boundary, Proc. Amer. Math. Soc. 63 (1977) 273-280.

10. M. J. Moore, Fixed points of automorphisms of compact Riemann surfaces, Can. J. Math. 22 (1970) 922-932.

11. S. M. Natanzon, Automorphisms of the Riemann surface of an $M$-curve, Funct. Anal. and Appl. 12 (1978) 228-229.

12. R. Preston, Projective structures and fundamental domains on compact Klein surfaces, (Ph.D. thesis. Univ. of Texas, 1975).

13. D. Singerman, On the structure of non-euclidean crystallographic groups, Proc. Cambridge Phil. Soc. 76 (1974) 233-240.

Departamento de Geometría y Topología

Facultad de Ciencias Matemáticas

Universidad COMPLUTENSE

MADRID, SPAin 